\title{
Rotordynamics Design and Test Results for a Model Scale Compulsator Rotor
}

\author{
Brian T. Murphy, Jon R. Kitzmiller, Ray Zowarka, Jon Hahne, and Alan Walls \\ The University of Texas at Austin Center for Electromechanics
}

\begin{abstract}
The model scale compulsator is a high speed (12,000 rpm), high energy rotating machine. The rotor is a highly optimized pulsed power electrical machine consisting of electrical windings, slip rings, and highly pre-stressed composite bandings. This paper describes the design of this machine from the standpoint of rotordynamics.

The rotor is supported on oil-lubricated hybrid ceramic duplex ball bearings, which in turn are supported on compliant squeeze film dampers. Test results are presented for both mechanical checkout runs and full energy discharge experiments. Also described is experience gained from low speed balancing on a commercial balancing machine, followed by high speed in situ balancing.
\end{abstract}

\section{INTRODUCTION}

A COMPULSATOR rotor is built to perform essentially two tasks: 1) store energy inertially in the mass of the spinning rotor and 2) rapidly deliver large portions of this energy in the form of a pulse of electric current. The design of the rotor is a challenging exercise in optimization as one strives to meet a wide variety of design goals and constraints. The compulsator discussed in this paper (Fig. 1) must be able to function in a mobile environment (i.e., on a vehicle, although not necessarily while moving). The rotor must have sufficient inertia to store a required amount of energy at a reasonable speed of rotation, while at the same time being as small and as light as possible. The rotor must accommodate electrical conductors and slip rings. Due to the nature of pulse power generation, the rotor must withstand potentially large impulse forces and torques during discharge.

To perform its basic function as a rotating machine, the compulsator rotor must be supported on radial bearings. The bearings must possess adequate load capacity to handle discharge transients, and must also allow the rotor to operate smoothly with sufficient life at high speed. Smoothness of operation is dictated by the precision of balance achieved by balancing the rotor on a commercial balancing machine or within its own housing, or by using both methods.

All rotating machines also have resonance speeds called critical speeds. These are speeds where vibration levels reach their maximum values due to the close proximity of natural

Manuscript received December 21, 1999. Funding for this effort was provided by the U.S. Army under contract DAAA21-92-C-0105. The COTR was Dr. Ed Schmidt. modes vibration. An important aspect of compulsator design is minimizing the potential for high vibration when operating through (or on) a critical speed.

\section{ROTOR DESIGN AND ANALYSIS}

Many of the issues regarding rotordynamic design for compulsators have been published previously [1]. For the model scale compulsator discussed in this paper, The University of Texas Center for Electromechanics (UT-CEM) employed the same design approach which has been used successfully in the past. The rotor structure (Fig. 1) is designed to be relatively short and stiff. "Relatively short" means that the length-to-diameter ratio of the main body of the rotor is not greater than approximately two. The meaning of "relatively stiff" is with respect to the frequency of rotation at maximum speed (200 Hz in this case). A rotor which is "stiff" has its first free-free mode of lateral vibration (i.e., bending) well above the maximum frequency of rotation.

Fig. 2 shows the finite element rotordynamic model of the subscale compulsator. This model is analyzed with an inhouse developed program (TXROTOR) created specifically for rotordynamics. The model employs three node isoparametric beam elements [2]. The dynamic analysis takes into account gyroscopic effects due to rotor spin [3]. When the rotor is not spinning, the shaft can vibrate in a single plane. For example, Fig. 3 shows the fundamental bending mode where the rotor is flexing side to side in a horizontal plane. This mode shape could be measured just as it appears in Fig. 3 by conducting a rap test. In conjunction with this mode, there is an analogous orthogonal mode with the same frequency, flexing up and down in a vertical plane. These horizontal and vertical modes form what is referred to as a pair of planar modes.

When the rotor is spinning, gyroscopic action causes this pair of planar modes to become a pair of whirling modes. Fig. 4 shows a whirling mode of the compulsator rotor while the rotor is spinning at $12,000 \mathrm{rpm}$. This mode corresponds directly to the non-spinning mode in Fig. 3. In Fig. 4, if the direction of whirl is the same as rotor rotation, the mode is termed a "forward" mode. The counterpart in this pair of modes whirls in a direction opposite rotor rotation, and is termed a "backward" mode. The rotor must be spinning to enable the distinction between forward and backward modes.

It is gyroscopic action that causes the modes of the spinning rotor to be whirling instead of planar. More importantly, however, gyroscopic action also affects the 
frequencies of modes. One of the most important phenomena in high speed rotating machinery is that the frequencies of forward modes increase as shaft speed increases. In contrast, frequencies of backward modes decrease as speed increases. Fig. 5 shows how the natural frequencies of vibration of the model scale compulsator vary with rotational speed. Because the rotor is stiff, and the supports are soft (see below), the first two modes of the rotor will be primarily rigid body modes - one a bounce mode (Fig. 6) and the other a conical mode (Fig. 7). The frequency of the bounce mode does not vary with speed because gyroscopic action results from tilting of the rotor. Since the bounce mode has little to no tilting, there is no significant gyroscopic effect for this mode. The conical mode has much tilting, so gyroscopic action causes appreciable variation in its frequency with rotor speed. The first flexible bending mode (Fig. 4) of the model scale rotor is predicted to be over $30,000 \mathrm{cpm}$. Since this is so much higher than the 12,000 rpm maximum operating speed, it is not included in Fig. 5.

As in earlier compulsator designs, the model scale compulsator is supported in rolling element bearings. A duplex pair of angular contact ball bearings is used at each end of the rotor. One pair is mounted to provide radial support only, and the other provides both radial and axial support. Such bearings have very high mechanical stiffness, but not high enough so that the model scale compulsator can rotate up to $12,000 \mathrm{rpm}$ without traversing at least one resonant critical speed. For this reason the ball bearings which support the rotor are in turn supported in squeeze film dampers (SFD). Reference [1] presents details of the bearings and SFDs used on the model scale compulsator rotor.

Optimal stiffness and damping properties for the SFDs were initially selected so that the bounce mode would be traversed at 3,500 rpm, and the conical mode at 6,500 rpm. This would place both of these modes outside normal operating speed range of the model scale compulsator $(8,000$ to $12,000 \mathrm{rpm})$. The damping of the SFDs was selected to limit vibration response adequately while traversing these modes. When the SFDs were fabricated for this project, however, their stiffness and damping properties were both considerably higher than the selected optimum values, resulting in predictions of the bounce mode at $6,800 \mathrm{rpm}$ and the conical mode at $10,600 \mathrm{rpm}$. The damping provided by the SFDs was predicted to be adequate to limit vibration to acceptable levels; therefore, the nonoptimal SFDs were used without modification. As a result, the model scale rotor has a decreased tolerance to rotating mass imbalance, which means that while running with these particular SFDs, the rotor will require a more precise balance than would otherwise be required for smooth operation.

\section{BALANCING}

Balancing of the model scale compulsator is primarily a two step process: a low speed balance followed by a high speed balance. All balance corrections are made by inserting screws at two axial planes, one at each end of the main body of the rotor. Initially the rotor is balanced at a low rotational speed (around $700 \mathrm{rpm}$ ) with a commercial balance machine using industry standard methods [4]. Except for the absence of a coupling hub, the rotor is fully assembled and spinning in its own ball bearings during low speed balancing on a balance machine. After initial fabrication of the rotor, the residual mass imbalance was measured to be approximately $0.13 \mathrm{~mm}$ (0.005 in.) of mass center offset. The low speed balance operation reduces this offset to around 50 to $75 \mathrm{~nm}$ ( 2 to 3 $\mu$ in.).

When the rotor spins at high speed, nonuniform centrifugal growth of an elastic nature causes the balance to change. Since this change does not occur during the low speed balance, a high speed trim balance can be performed to reduce vibration experienced at high speed. The balance correction required to accomplish this amounted to approximately $4 \mu \mathrm{m}$ (0.00015 in.) of mass center offset. This means that as the rotor stretches while spinning up to 12,000 $\mathrm{rpm}$, the mass center moves relative to the bearing centerline approximately $4 \mu \mathrm{m}$ (0.00015 in.).

The high speed trim balance is conducted in a two step fashion. The first step is to determine and install a static trim balance (i.e., equal balance weights in each balance plane as shown in Fig. 8). This gets the rotor running smoothly while accelerating through the bounce mode critical speed. Then at the higher speed of the conical mode, a couple trim balance is determined and installed (i.e., equal and opposite balance weights in the two balance planes as shown in Fig. 8).

\section{TEst Results}

Fig. 9 shows a sample of vibration data for the model scale compulsator when first run after the low speed balance, but before any trim corrections were installed. Even with the excellent balance achieved with the low speed balance machine, vibration is still appreciable at high speed due to nonuniform centrifugal growth of the rotor. The data indicates that the critical speed is $9,200 \mathrm{rpm}$. This speed is considerably higher than the expected critical speed of 6,500 rpm mentioned earlier. The predicted 6,500 rpm critical speed was also predicted to be heavily damped, to the extent that it should not be very noticeable in the vibration data. Close inspection of the data in Fig. 9 shows that there is a modest rise in vibration near 6,500 rpm. The high vibration that peaks at 9,200 rpm is primarily due to vibration of the machine housing. Accelerometers mounted on the housing near the two bearing locations show that the machine is vibrating in a bounce mode fashion. A more detailed operating deflected shape of the housing vibration has not been measured or calculated as of this writing.

Using the influence coefficient method of balancing [4], which requires one run with a trial weight, a static trim correction was added to the rotor equivalent to $3 \mu \mathrm{m}(0.00013$ in.) of offset. In Fig. 9 the data shows that the vibration was greatly reduced at the critical speed by the static trim correction.

At speeds above 10,000 rpm, vibration starts to increase again as the conical mode is approached. A second high speed balance was then performed, this time to add a couple trim weight. This trim further reduced the vibration, thereby 
allowing the rotor to run smoothly up to a maximum planned speed of 12,200 rpm.

The procedure of trimming the rotor's high speed balance was carried out successfully on multiple occasions during commissioning of the machine.

Fig. 10 shows vibration data for all runs to $12,000 \mathrm{rpm}$ which have been conducted to date. The first run was a mechanical checkout run without an electrical discharge. Three subsequent tests all included high energy discharges from 12,000 rpm. Throughout this series of tests the rotor vibration remained extremely low (less than $1 \mathrm{~g}$ up to 12,000 rpm. No further trim balancing was performed. The vibration traces in Fig. 10 indicate a modest degradation in the static balance, as evidenced by the increasing vibration level near 9,000 rpm. Apparent changes in the state of couple balance were even less, where vibration is seen to decrease slightly in the 11,000 to $12,000 \mathrm{rpm}$ speed range. Considering the enormous mechanical and thermal shocks to which the rotor is subjected during discharge, the robust performance demonstrated by the model scale compulsator is considered excellent.

\section{CONCLUSIONS}

The design and testing of a model scale compulsator rotor have been described. Valuable experience was gained during the balancing of this rotor. The rotor was first balanced to extreme precision at low speed on a commercial balancing machine. At higher speeds, non-uniform elastic centrifugal growth necessitated a high-speed in situ trim balance to achieve the same precise state of balance. After high-speed balancing, the rotor was found to maintain adequate balance even throughout a series of high energy discharge experiments at the maximum rated design speed.

This highly successful test program is very encouraging from a mechanical design standpoint. In spite of operating the machine with nonoptimal squeeze film dampers, the vibration performance demonstrated was excellent.

\section{REFERENCES}

[1] B.T. Murphy, S.M. Manifold, "Compulsator Rotordynamics and Suspension Design," 1995 EML Conference.

[2] K-J. Bathe, Finite Element Procedures In Engineering Analysis, Englewood Cliffs, N.J. : Prentice Hall, 1995

[3] J.M. Vance, Rotordynamics of Turbomachinery, John Wiley and Sons, New York, 1988.

[4] N.F. Rieger, Balancing of Rigid and Flexible Rotors, Shock and Vibration Information Center, Naval Research Laboratory, Code 5804, Washington, DC, 20375-5000.

\section{FIGURES}

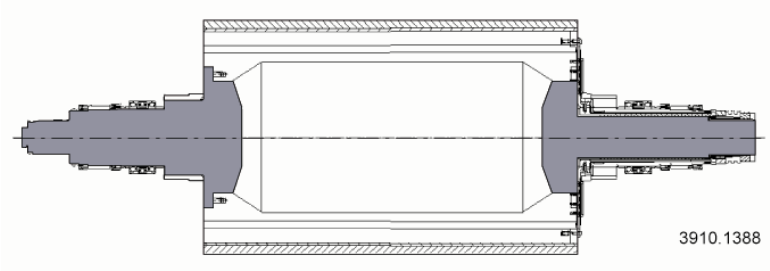

Figure 1. Model scale compulsator rotor.

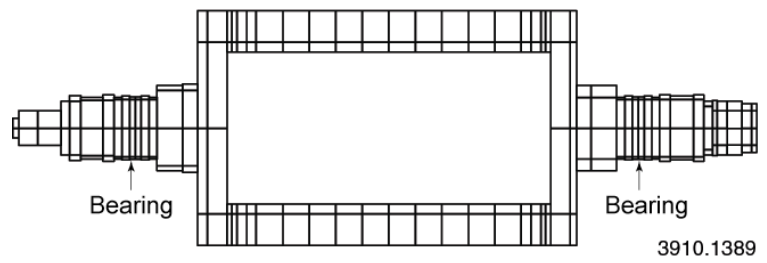

Figure 2. Finite element model geometry of model scale compulsator rotor.

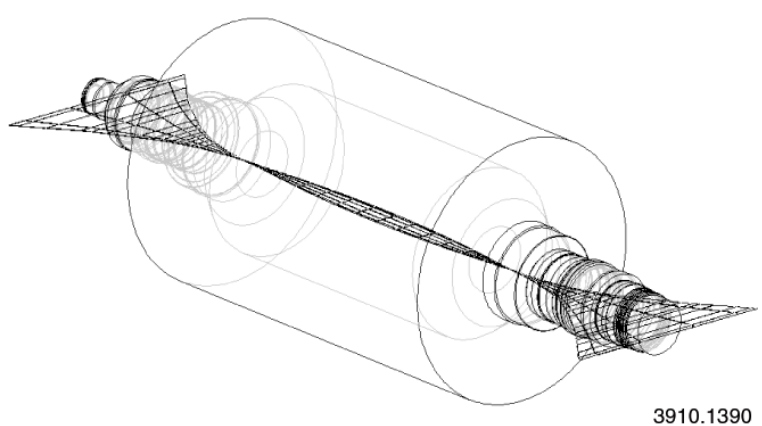

Figure 3. Fundamental bending mode shape of model scale compulsator rotor at zero speed. 


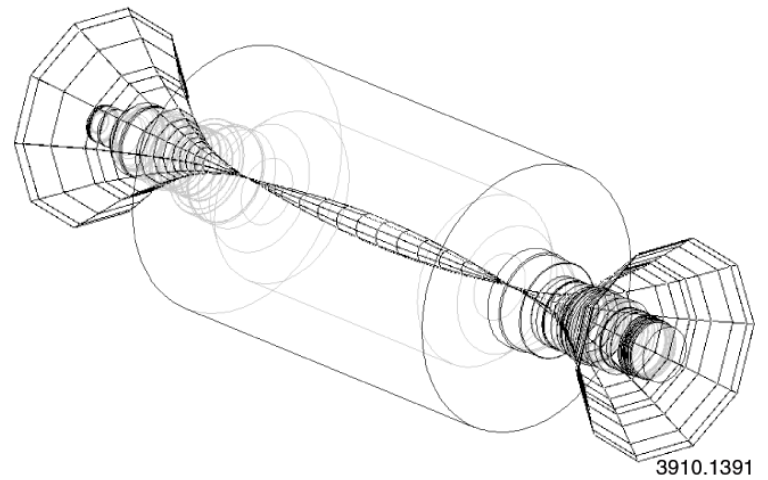

Figure 4. Fundamental bending mode shape of model scale compulsator rotor at $12,000 \mathrm{rpm}$.

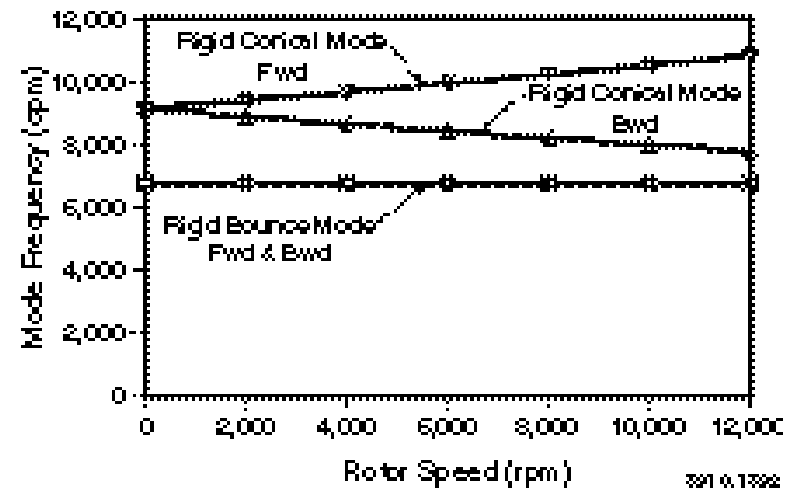

Figure 5. Damped natural frequency map of model scale compulsator rotor.

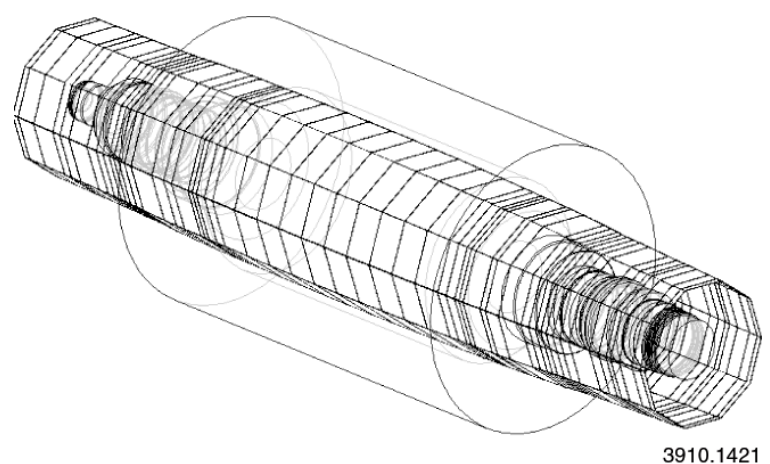

Figure 6. "Bounce" mode shape of model scale compulsator rotor at 12,000 rpm.

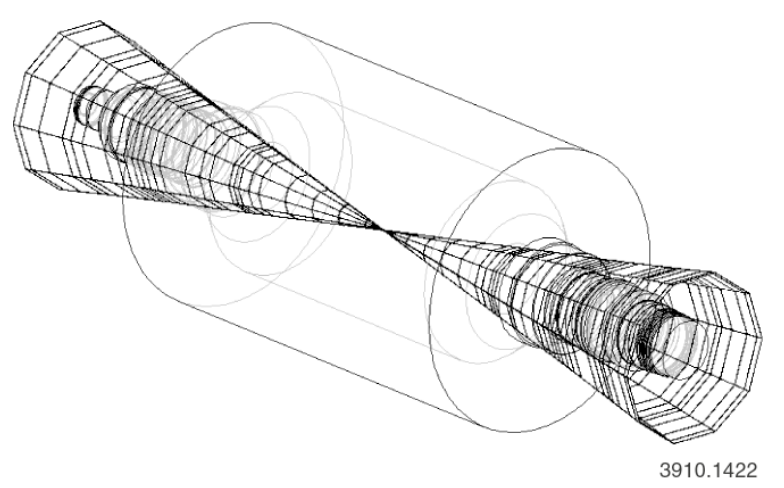

Figure 7. "Conical" mode shape of model scale compulsator rotor at 12,000 rpm.
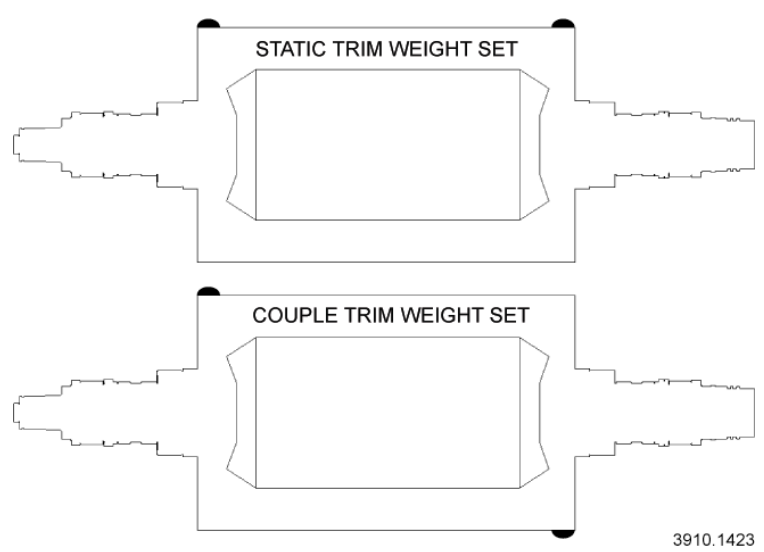

Figure 8. Location of balance weights for static (top) and couple (bottom) trim balance corrections.



Figure 9. Vibration data for the model scale compulsator rotor both before and after high speed trim balancing. 


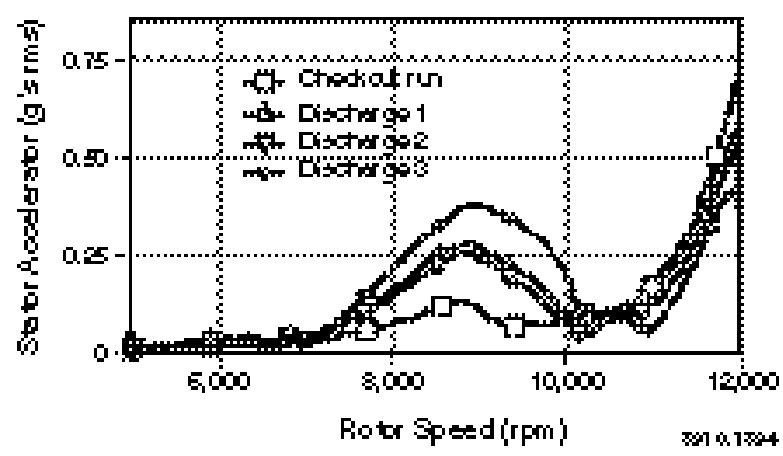

Figure 10. Vibration data for the model scale compulsator rotor for all runs conducted to date to its maximum design speed of 12,000 rpm. 